\title{
Conceptions of Infinity and Set in Lorenzen's Operationist System
}

\author{
Carolin Antos
}

\begin{abstract}
In the late 1940s and early 1950s, Lorenzen developed his operative logic and mathematics, a form of constructive mathematics. Nowadays this is mostly seen as a precursor of the better-known dialogical logic, ${ }^{1}$ and one might assume that the same philosophical motivations were present in both works. However, we want to show that this is not everywhere the case. In particular, we claim that Lorenzen's well-known rejection of the actual infinite, as stated in Lorenzen 1957, was not a major motivation for operative logic and mathematics. Rather, we argue that a shift happened in Lorenzen's treatment of the infinite from the early to the late 1950s. His early motivation for the development of operationism is concerned with a critique of the Cantorian notion of set and with related questions about the notions of countability and uncountability; it is only later that his motivation switches to focusing on the concept of infinity and the debate about actual and potential infinity.
\end{abstract}

\section{Introduction}

In his work on the philosophy of mathematics, Paul Lorenzen was motivated by the so-called foundational crisis of mathematics and the challenges mathematics was confronted with at the beginning of the 2oth century. ${ }^{2}$ To

\footnotetext{
Carolin Antos

University of Konstanz, Germany, e-mail: carolin.antos-kuby@uni-konstanz.de

${ }^{1}$ Notable exceptions are the works of Schroeder-Heister (2008), Coquand and Neuwirth (2017) and Kahle and Oitavem (2020).

${ }^{2}$ At least this pertains to his broader philosophical motivations. In the beginning, mathematical reasons were more prominent, as Lorenzen recognized the impact of his work in lattice theory on consistency proofs (see Coquand and Neuwirth 2017; Coquand and Neuwirth 2020). I would like to thank one of the referees for pointing this out to me.
} 
tackle the problems raised during this crisis, he developed in the 1950s a new foundational system for mathematics, a system he called "operative logic" and "operative mathematics". ${ }^{3}$ Nowadays, the work on operative logic is mostly referred to as a precursor of his much better known later work on dialogical logic. Indeed, this approach was developed as an answer to the shortcomings of operative (proto-)logic. ${ }^{4}$ In this article, we want to focus on his operative mathematics, which is an elaborate attempt at building a constructive version of mathematics that still preserves (most of) modern analysis. Although he later abandoned the specific setup of operative mathematics, he continued to pursue the general ideas and motivations operative mathematics rested on.

At the beginning of the 1950s, however, Lorenzen still perceived his operative logic and mathematics to be nothing less than a "new way to overcome the foundational crisis" (Lorenzen 1956b). ${ }^{5}$ This new way is situated between the two main factions that developed answers to the crisis, the "Hilbertians" and the "intuitionists". In Lorenzen's view, the question of a "good" foundation for analysis remained unanswered by both accounts and so he sought out a new way towards a foundation by using methods and ideas of both approaches while overcoming the problems they were faced with. Central to this endeavor is a thorough treatment of the real numbers. According to Lorenzen, it was the (mistaken) treatment of the reals that led to the development of Cantorian set theory and the connected notion of mathematical infinity, which in turn gave rise to the problems that were at the heart of the foundational crisis.

Nowadays, Lorenzen is mostly known for his rejection of actual infinity. Indeed, Lorenzen (1957) claims that the next big challenge for mathematics is to show that "the infinitely large (more precisely, the actual infinite) is to be demonstrated to be disposable". ${ }^{6}$ Therefore, it might seem as if the rejection of actual infinity had also been a central motivating factor for his development of operative mathematics.

In this article, we want to argue that this is in fact not the case. Instead, we claim that the rejection of actual infinity was not prominent in his development of operative mathematics; in particular, the notion of infinity was not his main focus, but rather the notion of set was. Our hypothesis is that a shift occurred in Lorenzen's treatment of infinity: in the beginning he

\footnotetext{
3 A complete presentation can be found in Lorenzen 1955. For all quotes from this book and all the other German texts, the translations into English are my own.

4 For a detailed account of how and why Lorenzen abandoned operative logic and developed dialogical logic, see Lorenz 2001.

5 "Die operative Logik und Mathematik stellt einen neuen Weg der Überwindung der Grundlagenkrise dieser Wissenschaften dar." Lorenzen 1956b, a short but very informative outline of the main ideas of his operationist system, also sketches a larger project he seems to have had in mind, by planning on exploring the ramifications operative mathematics has for wider applications in the sciences.

${ }^{6}$ Lorenzen 1957, 11; translation taken from Lorenzen 1987, 202.
} 
focused on the notion of set and, connected to this, the notions of countable and uncountable sets; ${ }^{7}$ only in the late 1950 os did his focus shift towards the question of potential and actual infinity. ${ }^{8}$

To make this hypothesis plausible, we will first give an overview of how Lorenzen's operative approach is embedded in the general discussion of the foundational crisis in mathematics and, in particular, how it relates to the major approaches of formalism and intuitionism (Section 2). To explain the first part of the proposed shift in Lorenzen's work, we will take a closer look at how Lorenzen eliminates the classical notion of set and how this impacts the notions of countability and uncountability in operative mathematics (Section 3). In Section 4 we will compare his treatments of the notion of infinity from the early and late 1950 os and argue for the shift in Lorenzen's treatment of infinity. In the last section, we will suggest possibilities for future work on the explanation of why this shift occurred and the systematic question whether operative mathematics is a valid framework for potential infinity.

\section{Operationism and the foundational crisis}

Before going into the details of how Lorenzen attempted to tackle the question of the notions of set and infinity in an "operative" way, we want to show how Lorenzen situated his approach in the discussion about the foundational crisis in mathematics. This chapter therefore also serves as a short overview of Lorenzen's thought on the foundations of mathematics in the 1950s, as most of his work from this time is quite unknown to an international audience. ${ }^{9}$

In the late 1940s and early 1950s, Lorenzen was working on a constructive account of mathematics that had at its center the notion of operations via certain schematic rules. In Fraenkel, Bar-Hillel, and Levy 1973, it is described as follows:

For Lorenzen, the main (though not the only) subject of mathematics is the treatment of calculi - this should by no means be misunderstood as a claim that mathematics is a calculus, which Lorenzen would very definitely reject where a calculus is understood to be a system of rules for schematic operations

7 A set $X$ is countable if there is an injective function from $X$ to the set of the natural numbers; $X$ is uncountable if there is no such function.

8 To explain what these terms mean, Lorenzen (and others) often point to Aristotle's definition in his Metaphysics, book 9, chapter 6. Mathematical definitions of these concepts vary; they usually contain a description of some "construction process", but differ in how far the construction should proceed and whether this is spelled out via induction, computation or other approaches. In Subsection 4.2 we discuss the possibilities of interpreting Lorenzen's notion of construct as a definition of potential infinity.

${ }^{9}$ One reason for this is that most of his papers were published in German and no English translations are available. 
with figures, which may but need not be marks on paper; they might as well be pebbles (calculi) or any other physical objects. In addition to this precisification of the subject-matter of mathematics - and only slightly connected with it Lorenzen stipulates that the methodical frame be as wide as compatible with the conditio sine qua non that all mathematical statements be definite. ${ }^{10}$ (p. 179)

Lorenzen acknowledges several sources for the ideas he uses in this operative approach, the most important among them being Weyl (1918). His approach is quite comprehensive, giving not only a new way of building up different areas of mathematics (with analysis as the most important one), but complementing this with a new logical system, operative logic, which in turn is founded on a so-called protologic.

He sees this as a kind of reverse account to Hilbertian mathematics and metamathematics: ${ }^{11}$

After it has become clear that an axiomatization of the naive theories is not enough, but is in need of a metamathematics, the task arises to justify the metamathematical modes of inference. The object of metamathematics are certain formal systems [Kalküle], viz., axiomatized theories. In an exact reversion of this line of research, in "operationism" it is the formal systems (i.e., operating with symbol strings [Figuren] as such) which are put at the beginning, metamathematics is thus supplanted by protologic. ${ }^{12}$ (Lorenzen 1956b)

In Lorenzen's view, Hilbert's Program and the distinction between mathematics and metamathematics are unable to adequately address the problem of a foundation for mathematics, for very basic, structural reasons:

According to Hilbert's Program for the foundation of mathematics, the task to provide consistency proofs is assigned to metamathematics.... As every proof is only as good as the methods it uses, it can be objected to such proofs that the inference by virtue of content [inhaltliches Schließen] is neither formalized nor indeed justified.

In attempting such a formalization it became apparent that the distinction between mathematics and metamathematics is not suited for the problem of foundations. The proof methods in metamathematics are none other than those

\footnotetext{
${ }^{10}$ For an example of such a system of rules, see the system $Z$ below; for a definition of definiteness, see Subsection 4.2.

${ }^{11}$ Note that Lorenzen's view on other ways out of the foundational crisis, as presented, for example, by Hilbert or Brouwer, are only described here insofar as they serve as a demarcation line for his operationism. In his later work, for example, Lorenzen 1960, 119, he did indeed argue that the foundational crisis has been overcome precisely because we have a fruitful interplay between Hilbert's and Brouwer's approach.

12 "Nachdem deutlich geworden ist, daß eine Axiomatisierung der naiven Theorien nicht genügt, sondern noch eine Metamathematik erfordert, stellt sich das Problem, die metamathematischen Schußweisen zu begründen. Gegenstand der Metamathematik sind gewisse Kalküle, nämlich die axiomatisierten Theorien. In genauer Umkehrung dieser Untersuchungsrichtung werden im ,Operativismus` beliebige Kalküle (also das Operieren mit Figuren als solches) an den Anfang gestellt, an die Stelle der Metamathematik tritt so eine Protologik."
} 
used in mathematics. A foundation of metamathematics is therefore nothing but the foundation of a part of mathematics .... ${ }^{13}$ (Lorenzen 1951b, 162)

So, instead of justifying the use of an axiomatic system in metamathematics (for example, by providing a consistency proof) in what could be perceived as a justification after the fact, Lorenzen puts the justification at the beginning of his investigation, via the protological principles:

One can, without assuming logical or mathematical knowledge, obtain certain protological principles which are sufficient to establish customary logic and mathematics .... (Lorenzen 1956b)

These basic principles are, for example, operations on symbol strings that have their origin in basic practices like pre-mathematical forms of counting. They give rise to formalized operations or rules through which the usual objects in mathematics can be defined. As one example, if one thinks about numbers in the manner of "strokes on paper" of the form $|,\|\||,, \ldots$, this can be written down as rules for manipulating symbol strings such as the following rule system $Z$ :

1. Begin with $\mid$.

2. If you have reached $x$, add $x \mid$.

So, general rules for how to operate with symbol strings are given via formal systems which then constitute the objects of operative mathematics. In this way Lorenzen can utilize an axiomatic methodology and at the same time have a sound foundation in virtue of the protological principles. ${ }^{14}$

Such an approach shows the importance of action and applicability in mathematics that is characteristic for Lorenzen's thoughts:

Such rules are not examined as to whether they are "true" or not-they are only examined as to whether they are "useful" or not, i.e., whether acting on these rules, meaning the construction of symbols, is suitable to some purpose. This, however, is no longer a mathematical question, but belongs to applications. (Lorenzen 1951b, 163)

This applicability is also the biggest difference between Lorenzen's operative mathematics and intuitionistic approaches like Brouwer's and Weyl's. For Lorenzen, a good foundation of mathematics should preserve the full power of contemporary analysis. This means, in particular, that one should be able to use tertium non datur, which is made possible in operative mathematics:

13 Of course this is only Lorenzen's account of Hilbert's Program, and it would be interesting to see if it is a faithful one. For a non-Lorenzen viewpoint, see, for example, Sieg 1999.

14 Lorenzen was not opposed in general to using axiomatic methods, as long as they were not used as a foundation: "Axiomatizations have no other purpose than to simplify operating on constructible sets, functions, real numbers etc." (Lorenzen 1951b, 165). Lorenzen $(1960,118)$ calls this the "systematic priority of axiomatic mathematics over constructive mathematics." 
In contrast to intuitionistic attempts, we are now-after an unobjectionable foundation of logic-allowed to always use the tertium non datur. The uncomfortable restriction to "decidable properties", "enumerable real numbers", etc. is no longer necessary. An attempt in this intended direction was made already by Weyl in 1918-however, it had to fail, because a justification of the tertium non datur was still lacking. (Lorenzen 1951b, 166)

Lorenzen addresses this issue in operative logic by distinguishing between the effective predicate calculus (effektiver Quantorenkalkül) (which is in essence the intuitionistic approach) and the fictional predicate calculus (fiktiver Quantorenkalkiul), which includes the tertium non datur and is therefore the classical predicate calculus. As the word "fictional" suggests, the justification of such a calculus means to "justify a fiction" (Lorenzen 1955, 79). This justification can be given "in most of the cases" (Lorenzen 1955, 84) by a careful analysis of the concepts of effective and fictional derivability or underivability. Using these means, Lorenzen achieves a compatibility of operative analysis with classical analysis-although he notes that " $[t]$ he operative conception also implies that the intuitionistic opinion is right in that 'in fact' only effective derivability is of interest" (Lorenzen 1955, 84).

Operative logic and mathematics is deemed to be a third way out of the crisis in that it overcomes both the foundational weakness of formalistic approaches and the lack of full applicability of the intuitionistic approaches, while at the same time borrowing some of their strengths. Incidentally, this also holds for Russell's solution to the crisis. As we will see in the next section, Lorenzen uses a version of Russell's ramified type theory to avoid impredicativity:

With the laudable exception of the intuitionists, who went their own way, this approach (Russell's ramified type theory [verzweigte Stufenlogik]) has been abandoned in favor of unramified type theory only because ramified type theory was allegedly too complicated, because the stock of modern mathematics could not be "saved" in a satisfactory manner. However, the impredicativity of this logic has never been justified, at least until now: the error of infinite regress cannot be avoided this way. Yet ramified types [verzweigte Stufen] can be much simplified by introducing something like untyped branches [ungestufte Zweige], instead of appealing to unramified types .... And, with this, one remains "predicative" ... (Lorenzen 1956a, 275)

Contrary to the relative unrenownedness of Lorenzen's operationism in today's literature, ${ }^{15}$ the book was well received by his contemporaries. Nevertheless, it also met with criticisms, which mainly pertained to the foundational aspect of operationism: As protologic carries much of the foundational load, it is not surprising that in the reception of Lorenzen's work a great part of the criticism was directed against the protological principles and the claim that they give a sound foundation. William Craig singled this out as the main problem of Lorenzen's approach:

The main weakness of the book is its failure to indicate as clearly as a work on foundations should the strength of the methods employed and thus of the

15 A notable exception is Fraenkel, Bar-Hillel, and Levy 1973. 
underlying assumptions. Claims that only the principles of the chapter on Protologic are employed and that no understanding of other logical notions is required are unconvincing and seem unnecessary. (Craig 1957, 319)

Gerhard Frey $(1957,633)$ even claims that when asserting that mathematical and logical knowledge are not needed for operating with symbol strings, Lorenzen "circumvents ... the actual philosophical questions on purpose".

According to Lorenz (2001), Lorenzen himself was convinced to abandon operative (proto-)logic after a discussion with Tarski when he was visiting the Institute of Advanced Study in Princeton in the Fall 1957/58. Then, he began to develop what is now known as dialogical logic. But this rethinking of the foundations did not diminish the significance of Lorenzen's mathematical work. Thoralf Skolem points this out by writing in his review that "although one may doubt whether Lorenzen's theory is the best conception of mathematics, the reviewer believes that the book will have a sound influence on the mathematical world" (Skolem 1957, 290). This is also the essence of Wolfgang Stegmüller's extensive review of Lorenzen's book:

In particular the part that in the reviewer's eyes represents the most important contribution of Lorenzen towards a foundation of mathematics, namely the theory of the real numbers, is described in detail in this book; in fact, the thoughts developed in it are for the most part independent and can therefore be isolated from the operative framework of the theory and transferred to different system constructions. This point is not to be underestimated in an overall assessment of Lorenzen's achievement; as whatever one's opinion of Lorenzen's operative interpretation of logic and mathematics may be-his foundation of analysis is in most parts independent of this interpretation and will without doubt be inspiring and fruitful for all future attempts in this direction. (Stegmüller 1958, 161-162)

Despite his above-mentioned critique, Craig also concurs with Lorenzen's claim that the operative approach signifies a third way out of the crisis in much the same way as described above: "The reviewer believes that the book ... presents a legitimate and probably fruitful third approach to foundations. Its evident advantage over intuitionism is the preservation of classical logic and arithmetic and of much larger portions of the rest of mathematics, and over formalism the interpretation of these" (Craig 1957, 318).

\section{Elimination of the classical notion of set}

In the second half of the 19th century, set theory was developed in search for a sound foundation of analysis (see Ferreirós 2007). As is well known, the first approach, so-called "naive" set theory, led to paradoxes that were then addressed by a more appropriate axiomatization. Nowadays the ZermeloFraenkel axiomatization with the Axiom of Choice (ZFC) is considered standard in set theory. 
But for Lorenzen this whole development is unsatisfactory: Not only is an axiomatization not a valid kind of foundation for him, he believes that the whole concept of set that underlies Cantorian set theory is flawed. He makes this point in one way or another in nearly all of his papers concerned with foundational work. As he writes in the introduction to his 1955, 4: "In spite of Cantor's 'definition' of set-of which, as is known, nothing can be deduced, in as much as nothing can be deduced from Euclid's 'definition' of a point - a set in mathematics is never built through a 'collection into a whole' ...."

The fact that classical analysis rests on this (in Lorenzen's view) mistaken concept of set is one of the big challenges which analysis, and in turn modern mathematics, has to face. So, a central requirement for a valid foundation for mathematics is the elimination of the classical concept of set ${ }^{16}$ and its replacement with an alternative account of sets, while at the same time making sure that the fundamental theorems of classical analysis are preserved.

Lorenzen develops this alternative in a series of papers that are concerned with the concept of finite sets (Lorenzen 1952c), the concept of set and its use in topology (Lorenzen 1952a) and especially the concept of set in analysis (Lorenzen 1951a; 1951C). These papers lead up to Lorenzen 1955, where he explains in detail how the classical concept of set can be eliminated.

The core idea of Lorenzen's operationist system is the following: instead of having an informal definition of set that relies on a quasi-intuitive understanding of some kind of "collection", Lorenzen mathematically defines what a set is via abstraction from formulas. ${ }^{17}$ Formulas and relations themselves are defined by giving rules that produce them.

As an example for such a procedure, consider the way Lorenzen (1951a, 2) shows how unary relations are built for atoms $x, y, \ldots$ :

1. If $\sigma$ is a relation, then $\sigma(x)$ is a formula for a variable $x$.

2. Let $a, B, A(x)$ be formulas, then the following are formulas: $A \wedge B, A \vee B$, $A \rightarrow B, A \leftrightarrow B, \neg A, \forall x A(x), \exists x A(x)$.

3. If $A_{1}, \ldots, A_{\kappa}$ are formulas and $\sigma$ a relation, then the following is a relation:

$$
\mathbf{I}_{\sigma}\left(A_{1} \rightarrow \sigma\left(x_{1}\right), \ldots, A_{\kappa} \rightarrow \sigma\left(x_{\kappa}\right)\right) .
$$

Clause 3 means that $\sigma$ is the relation inductively defined by the rules

\footnotetext{
${ }^{16}$ For an instance where Lorenzen formulates this as a challenge, see Lorenzen 1954, 67.

17 For the purposes of this paper we will not differentiate between "Aussage" and "Aussageform", which we will both translate as "formula". The difference lies in the use of free variables (see Lorenzen 1955, 178).
} 


$$
\begin{aligned}
A_{1} & \rightarrow \sigma\left(x_{1}\right), \\
\vdots & \\
A_{\kappa} & \rightarrow \sigma\left(x_{\kappa}\right) .
\end{aligned}
$$

Over such a language, sets are now given through formulas: ${ }^{18}$

Let $A_{1}(x), A_{2}(x)$ be formulas such that, for all $x$,

$$
A_{1}(x) \leftrightarrow A_{2}(x)
$$

then $\mathrm{M}_{x} A_{1}(x)=\mathrm{M}_{x} A_{2}(x)$, where $\mathrm{M}_{x} A(x)$ is the set of $x$ such that $A(x)$.

Although the final setup in Lorenzen 1955 is more elaborate than this example, ${ }^{19}$ the basic idea remains the same: Operative rules tell us how to build up certain symbol strings which then are formulas, relations and so on. On this basis, objects like sets and functions can be built, and therefore precise definitions for them can be given.

Before we look more closely at how this setup enables Lorenzen to give an operative account of the real numbers, we will first consider the case of arithmetic. Here we can see in more detail how deeply the operative conception of set differs from the classical notion. For arithmetic, Lorenzen develops an account of how to treat finite sets, namely, not as a specific instance of the general concept of set, but rather as an object that can be treated independently from general sets:

\begin{abstract}
Since with the constructive foundation [of mathematics] one can already operate with symbols of arbitrary (finite) length before giving the definition of a formulaand therefore also before giving the definition of the notion of set-one would not want the notion of finite set to depend on the later choice of a definition of formula .... It is only the colloquial name "finite set" which misleads us into believing that one would have to define "set" as a basic concept and then "finite" as a specific difference. (Lorenzen 1952C, 331)
\end{abstract}

Consider a certain kind of symbol strings $x, y, \ldots$ that is built by writing the symbol strings $x$ and $y$ and so on in the given way (these symbol strings $x$ can, for example, be formulas of a certain calculus). Such an $x, y, \ldots$, called a system, can be derived from the following calculus:

${ }^{18}$ From the definition it may seem that there is no difference between Lorenzen's way of defining sets via abstractions as given here and (some form of) comprehension in standard set theory. However, Lorenzen had a more general notion in mind. When introducing abstractions in Lorenzen 1955, 100, to build terms from objects, he comments that starting with Frege and Russell one normally conceives of abstractions as classes:

With this, abstractions should be reduced to the introduction of "classes". However, we will see below that classes are nothing more than a special case of abstract objects. (Lorenzen 1955, 101)

${ }^{19}$ For instance, the defining schemata for relations have to satisfy certain criteria, such as foundation, to avoid circularity. 
I. $x \quad(x$ is a system $)$,

II. $\mathbf{r} \rightarrow \mathbf{r}, x \quad$ (if $\mathbf{r}$ is a system, then also $\mathbf{r}, x)$.

Finite sets can then be defined by abstraction: two systems yield the same finite set if they consist of the same objects, irrespective of the number and place of these objects in the system (so $x, x, y, z$ gives rise to the same finite set as $z, y, y, x, z)$, where the meaning of "consists of the same objects" is expressed through certain formulas. ${ }^{20}$ So, when considering finite and general sets, Lorenzen goes the exact opposite way to the classical approach: he first defines finite sets as separate entities and only later adjusts the process of abstraction for general formulas to arrive at general sets.

Indeed, he is able to set up the whole of arithmetic with the use of systems and finite sets only. For instance, numbers are defined via the system $Z$ from Section 2, and cardinal numbers are defined as the lengths of systems. In $\S 13$, where he defines basic numbers, Lorenzen includes two interesting philosophical comments. The first concerns the way in which operative arithmetic does not face the problem of incompleteness in the same way in which classical axiomatic approaches to arithmetic do. Because operative mathematics rests on protological principles, it is able to provide (operative) proofs "by reasoning in terms of content" (inhaltlich beweisen; Lorenzen 1955, 135) for sentences that would be undecidable in an axiomatic system because of Gödel's Incompleteness Theorem.

The second comment is one of the few times he explicitly refers to the underlying concept of infinity for operative mathematics in his 1955 . He points out that the system $Z$ that defines numbers contains the idea of iteration and therefore also the, as he calls it, "purest form of potential infinity" (Lorenzen 1955, 133). He assumes that this process of iteration or potential infinity, implicit in every rule of a calculus, is understandable to everybody. Note that this does not necessarily mean that he restricts himself to potential infinity or that he rejects actual infinity in operative mathematics. Instead, the reference to potential infinity has the purpose of explaining why the process of iteration meets the underlying requirements of protologic, ${ }^{21}$ namely to be understandable without assuming any prior logical knowledge. ${ }^{22}$

Of course, when considering analysis, finite sets are not enough. Rather, one has to use the general definition of sets via abstraction, as outlined above. Taking this as a starting point, Lorenzen then develops the so-called "language strata" (Sprachschichten), in which the objects of analysis appear step by step. The idea of language strata is intended to counter the underlying problem of impredicativity: Lorenzen $(1955,165)$ describes it, with Weyl, as the "mathematical process" that goes from considering things like

\footnotetext{
${ }^{20}$ Accounts of this way of treating finite sets can be found, for example, in Lorenzen $1952 \mathrm{C}$ and 1955 .

${ }^{21}$ See Section 2 for an example of the basic principles of protologic.

${ }^{22}$ We will discuss the question of potential and actual infinity in more detail in Section 4.
} 
numbers as objects to considering formulas about these objects as objects themselves - a process that in classical set theory allows the existence of the (unrestricted) power set. ${ }^{23}$

The language strata are built up in the following way (Lorenzen 1955, chap. 5): The basic language stratum $S_{0}$ consists of the basic objects, which can be built by operations on symbol strings. As we showed above, this also includes the basic numbers. $S_{1}$ then consists of all elements of the basic language and the first formulas that can be built via iteration of the following operations:

1. inductive definitions, which are usually formed through the use of an induction operator $\mathbf{I}_{\sigma}$ (Induktionsoperator), ${ }^{24}$

2. combinations with logical symbols $\rightarrow, \neg, \wedge, \vee, \forall, \exists$.

Sets of the first language stratum $S_{1}$ are then sets that can be formed in the way pointed out above via formulas of $S_{1}$. We can iterate this process and form $S_{2}$ in the same way we formed $S_{1}$ from $S_{0}$. In particular, we get a formula that represents an enumeration of the objects of $S_{1}$ and is itself an object of $S_{2}$. So, with $S_{2}$, we now have two types of sets: the sets that could already be formed from $S_{1}$ and the "new" sets that were formed by means of formulas from $S_{2} \backslash S_{1}$. This process can be iterated and gives us the sequence $S_{0} \subseteq S_{1} \subseteq S_{2} \subseteq \cdots$.

Interestingly, Lorenzen extends this method of iteration to countably infinite ordinals: $S_{\omega}$ is defined to be the union of the $S_{n}$ for all finite $n$ (and more generally, for a [countable] limit ordinal $\theta, S_{\theta}$ is the union of all $S_{v}$ for $v<\theta$ ). $S_{\omega+1}$ is then again built over $S_{\omega}$ by the usual iteration of inductive definitions and application of logical symbols. The question at which countable ordinal to stop this iteration process is open, ${ }^{25}$ though it is essential that it go beyond $S_{\omega} \cdot{ }^{26}$

In all language strata that are higher than the basic $S_{0}$ we are provided with iterations of sets: set of sets of basic objects, sets of sets of sets of basic objects, and so on; and if we want every set to appear as an element, we have to iterate up to a limit ordinal. At the same time, every higher language stratum also produces new sets of basic objects, as can be shown via Cantor's diagonalization method: there is no enumeration of the elements of $S_{v}$ in $S_{v}$, but there is one in $S_{v+1} \cdot{ }^{27}$ As a consequence, the power set becomes a relative notion, namely relative to a specific language stratum, where every higher language stratum adds more sets.

${ }^{23}$ As we have seen in Section 2, Lorenzen places himself in the tradition of Russell's type-theoretic approach (see also Lorenzen 1956a, 275).

24 We introduced this operator in the definition of a relation; see page 28 above.

25 Lorenzen 1955, 189, names $\omega+1,2 \omega, \omega^{2}$ and $\varepsilon_{0}$ as candidates; Lorenzen 1956a, 275, names $\omega^{\omega}$.

${ }^{26}$ Lorenzen $(1955,189)$ argues that "[f]or analysis, in the way we will develop it in chapter 6 , it is only necessary that we transcend $\omega . "$

27 For a detailed construction of this argument, see Lorenzen 1955, 191-192. 
In the same way we can also see that countability and uncountability are relative notions. As the enumeration of a language stratum $S_{v}$ is not an element of $S_{v}$, the set of all basic objects in $S_{v}$ is not countable in $S_{v}$, but it is in $S_{v+1}$. Therefore, uncountability will always be only relative to some language stratum, and this entails trivial solutions to the questions of the Axiom of Choice and the Continuum Hypothesis.

However, the difference between countability and uncountability is a central notion for classical mathematics, and therefore Lorenzen expands on the issue in Lorenzen 1956a. Here, he defines a substitute notion for uncountability and shows how it can be used to transfer the countability-uncountability distinction into operative mathematics. Consider the following definition (Lorenzen 1956a, 276): Fix a limit $\theta_{1}$ and a second limit $\theta_{2}>\theta_{1}$. All language strata up to $S_{\theta_{2}}$ are considered as constructed. Then we call all strata up to index $\theta_{1}$ "primary" and all higher strata up to $\theta_{2}$ "secondary". Similarly, a set is called "primary" if it is definable by a formula in a primary language stratum, and "secondary" otherwise.

Lorenzen immediately notes that the primary-secondary distinction does not seem to fit the usual countable-uncountable distinction. It can be shown that every infinite primary set contains secondary subsets, whereas subsets of countable sets are always countable. But, he argues, the notion of secondary set can be used in operative mathematics as a substitute for uncountability. To this purpose, he considers several examples from integration theory, measure theory and topology, in which instances he shows that the replacement works as intended. One example is the following: Real numbers are primary, as they can be introduced by dint of primary convergent sequences of rational numbers (which are primary themselves). But intervals of real numbers are secondary because every interval of reals consists of reals of arbitrarily high primary strata (Lorenzen 1956a, 276-277). He therefore argues that the notions of secondary set and uncountable set are "essentially" equivalent:

Summing up, I would like to claim that the use of secondary strata (obviously not without boundaries, only up to the unproblematic ordinal numbers, like $\omega^{\omega}$ ), in connection with the linguistic means [sprachliche Mittel] available in every stratum, gives us enough mobility to mostly follow the lines of reasoning [Gedankengänge] of modern mathematics. (Lorenzen 1956a, 279)

At this point Lorenzen developed an operative analysis, which is "in essence" (im wesentlichen) similar to classical analysis. By "classical analysis", Lorenzen means analysis "in virtue of content" (inhaltlich), so, not an axiomatic system of analysis but concrete, non-abstract analysis or-as he puts it-everything that can be found in "current textbooks" (Lorenzen 1955, 196). This includes in particular everything that is needed for the natural sciences, like theoretical physics. He has therefore achieved his main goals: to present a foundation for mathematics that on the one hand is meaningful by being based on a mathematically definable concept of set while on the other hand still preserving the essence of classical analysis. For our purposes, the former 
is the more informative conclusion. We can now state that the elimination of the classical concept of set was a main motivation for Lorenzen's work on operative mathematics and that, in his view, he achieved his goal of replacing it with a more acceptable one. In the next chapter, we will examine how this endeavor relates to Lorenzen's views on the concept of infinity.

\section{The question of infinity}

\subsection{A shift in focus}

Let us now return to our initial claim about a shift in Lorenzen's treatment of infinity. As we mentioned in the introduction, Lorenzen is known for his rejection of actual infinity in his 1957. There, he uses operative mathematics as an example for a foundation of analysis that uses only potential infinity instead of requiring actually infinite sets. He concludes with the statement that actual infinity should be eliminated from modern mathematics (Lorenzen 1957, 11).

This might suggest that operative mathematics was motivated by the search for a framework that allows for such an elimination. Indeed this claim is made in the literature: In Stegmüller's review of Lorenzen's book (1955) on operative mathematics, he remarks that "from the beginning, the author is concerned with allowing no more than the 'potential infinite' in the foundation of mathematics, ... while abandoning the idea of 'completed infinities'" (Stegmüller 1958, 177). Heyting (1957) reviews both papers 1957, 1954 at once. He judges the former as the philosophical and the latter as the more mathematical paper, which together explain Lorenzen's operationist system. He points out that "the author agrees with intuitionists in so far as he does not accept the notion of actual infinity in mathematics" (Heyting 1957, 368).

Both reviews, however, appeared after Lorenzen 1957 (in the case of Heyting that is of course necessarily the case). Prima facie this does not seem to be problematic, as all of these works were tightly linked by the common topic of the operationist approach and its foundational role, as well as by publication date. But, surprisingly, when reviewing these works not in retrospect, but in chronological order, as a single body of work, actual infinity is not explicitly rejected by Lorenzen until his 1957. Rather, in the development of his operationist system, the question of infinity is not prominent at all. As we have shown in the previous chapter, what is heavily discussed is the notion of set and the deficiencies of the Cantorian notion of set, which make it unsuitable as a "good" foundation for mathematics.

This leads us to propose that in Lorenzen's focus on foundational questions a shift occurred, from the notion of set to that of infinity. Let us give the following classification: 
First phase: In the early 1950 os Lorenzen focuses on the notion of set. He sees it as his central task to eliminate the classical notion of set and to replace it with a rigorous operative definition. This provides a solution of the problem of uncountable sets, as uncountability only remains in a relative version, whereas in an absolute sense, all sets are countable.

Second phase: In the late 1950s Lorenzen's focus is on the notion of infinity. This is now primarily framed as the distinction between potential and actual infinity and it culminates in the goal of eliminating the actual infinite from mathematics.

To make the time frame of the above classification a little more precise, we will count the articles that lead up to Lorenzen 1955, and papers that expand on this, like Lorenzen 1956a, as belonging to the first phase, whereas Lorenzen 1957 marks the shift towards considering the question of infinity as the more fundamental one and is therefore already representative of the second phase.

Let us point out more clearly what this shift does and does not imply: It does not imply that Lorenzen's topic of research changed between the two phases outlined above. Instead, it is a change of focus regarding the issue of which notion (set or infinity) is the more fundamental and, therefore, whether eliminating the one or the other gives us better reasons to argue that the mathematical outcome is free of the inherent flaws of other foundational approaches. The technical way of explicating such a good foundation can beand indeed is - the same, namely the framework of operative mathematics, but the reason for why one should consider it to be a good foundation differs. Naturally, the notions of set and of infinity are interconnected: in the first phase, the argument that the newly developed notion of set only relies on potential infinity is used as an additional argument for its soundness; in the second phase, the intended potentialist notion of infinity is explicated by using the operative notion of set. ${ }^{28}$ Nevertheless, it still matters strongly which of the two notions is regarded as basic for foundational purposes, not least because changing one does not have to entail changing the other. ${ }^{29}$

So, our main goal here is to show that such a shift in focus occurs in Lorenzen's work. However, in proposing the occurrence of such a shift, we make no claim on whether operative mathematics constitutes a potentialist framework regarding infinity or not. Indeed we will treat this as a separate question, which due to constraints of space and time will have to be considered in a different article. The same holds for the question of why such a shift occurs. An analysis of this would require a much wider consideration of Lorenzen's philosophical development than we are able

\footnotetext{
${ }^{28}$ Examples where Lorenzen argues along these lines will be given below.

29 The same holds for a connection that seems even more fundamental, namely, that between intuitionistic logic and potential infinity. Although it is generally assumed that they depend on each other, Linnebo and Shapiro (2019) show that there is an explication of potential infinity that still allows for classical logic.
} 
to present in this context. We will therefore limit ourselves to pointing out some considerations towards both questions at the end of this article.

In the rest of this article we will argue for Lorenzen's proposed change in focus in the following way: Having already described that the elimination of the Cantorian notion of set was a main motivation for the development of operative mathematics in the first phase (see Section 3), we will show how Lorenzen shifts towards regarding, in the second phase, the question of infinity as more fundamental. As this is spelled out quite clearly in Lorenzen 1957, the main burden of proof for the proposed shift lies in arguing that the question of infinity was not prominent as the main foundational motivation in the first phase.

Let us therefore start by addressing this point first. What we intend to show is that in the first phase Lorenzen places no special emphasis on the question of potential vs. actual infinity and indeed does not explicitly commit himself to a standpoint in this debate. We will present two arguments: first, we point out that Lorenzen simply does not explicitly reject actual infinity, even in cases where it would be obvious to do so; and second, we lay out his argument that the problems usually associated with infinity are in fact problems that arise because of the Cantorian notion of set, whereby he reduces the question of infinity to that of the notion of set.

\subsection{Constructs and infinity}

Both lines of argument can be seen quite clearly in his article "On the consistency of the concept of infinity" (Lorenzen 1952b). In the first sentence he states: "The problematic nature of the concept of infinity is independent from that of set" (p. 591). This may seem surprising at first, as one major motivation of operative mathematics is to address the problems in modern mathematics by eliminating the classical notion of set. But in the following it becomes clear that by "concept of infinity" he means something very specific, namely the notion of infinity as it appears in arithmetic: "If one poses the question of the consistency of the notion of infinity, one wants to know whether no contradictory statements can be proven over $\mathbb{N}$, i.e., whether arithmetic (the theory of $\mathbb{N}$ ) is consistent" (1952b, 591). He then explains this further by arguing why the natural numbers are the right choice for such an investigation into the concept of infinity:

Of course one could take any infinite set instead of $\mathbb{N}$-however, with such a formulation one would unnecessarily enter into the difficulty of the concept of set. What makes $\mathbb{N}$ so unproblematic as a set is that each of its elements is constructible. Starting from 1 , by constructing an additional $a+1$ to each $a$, one obtains all natural numbers. We will call every set a "construct" whose elements can be constructed in such a (eventually much more complicated) way. (Lorenzen 1952b, 591)

He elucidates this point in the conclusion of the article: 
In cases where the dangers of contradiction still exist in connection with an infinite set, e.g., the set of all real numbers, all real functions, as they are used in classical analysis - in all these cases one will need to find "the error" not in the concept of infinity, but in the concept of set. (Lorenzen 1952b, 594)

How is this presentation of a concept of infinity to be understood? Being aware of Lorenzen's decisive rejection of actual infinity in later articles, one could in retrospect read this as the difference between potential and actual infinity. Indeed, several points seem to support this thesis, at least when looking at the first term of the distinction, namely, potential infinity. The way in which "constructs" are introduced accords with the usual way in which potential infinity is explained, i.e., there is some kind of procedure that can be used again and again, with the possibility of going on without end. So, it could be that, for Lorenzen, constructs are an acceptable way of describing infinity because, by being the products of a well-defined process, they are compatible with the framework of potential infinity.

However, if we claim that, in restricting the concept of infinity to that of constructs, the underlying motivation derives from the actual-potential infinity distinction, this immediately raises the question: Why doesn't Lorenzen say so? Instead, Lorenzen never once uses the term "potential infinity" throughout the article. A similar point could be made for his treatment of actual-potential infinity in his book on operative mathematics (Lorenzen 1955). Here, he does mention both concepts already in the introduction, when he describes Brouwer's intuitionistic program $(1955,2)$. But even though he uses the introduction many times to point out which philosophical and mathematical thoughts have influenced him in his work, he never commits to a standpoint in the actual-potential infinity debate. And, as we have seen in Section 3, even when he mentions potential infinity later on in the book, he never goes so far as to explicitly reject actual infinity. One example for such a remark is: "We have already when dealing with protologic presupposed the capacity on the part of the reader to conceive of every rule of a calculus as something potentially infinite" $(1955,133)$. The maximal commitment we find here is that he does not have to stay in a finitist framework because of his assumption that agents carrying out the schematic operations have the capacity to work with the potentially infinite.

Still, this kind of argument does not seem sufficient. There could be a number of reasons why he never explicitly stated the connection to the actual-potential infinity debate: he might have considered it to be unimportant to make this philosophical point in a more mathematical paper; he might have regarded it as so obvious that there was no need to bring it up; he might have been unaware of his underlying motivations (though this is quite unlikely).

To settle the question more satisfactorily, let us look more closely at the notion of "constructs" he introduces in the paper 1952b. This term seems to capture the concept of infinity he wants to consider (because it is an acceptable concept of infinity), whereas problems arise with sets that are 
not constructs. So our question from above reduces to the problem whether the introduction of constructs is motivated by questions about infinity or about the concept of set. As we have seen above, an argument can be made that the definition of constructs is motivated by the concept of potential infinity. What, then, about actual infinity? Could it be that Lorenzen simply understands everything which is not a construct, for example, the set of the real numbers, as an actually infinite set and therefore rejects it as an instance of acceptable infinity? He seems to hint at something like this when talking about what he (later in the article) calls the "schism in mathematics":

\begin{abstract}
Whether it is justified to demand a ban of all those sets which cannot be reduced to constructs, that is still a debated question. If one leaves this question open, the interesting situation arises which is on display in current mathematics: there are actually two mathematics, a constructive one (in particular in the intuitionistic form) and a classical one (the axiomatic variety also belongs here). (Lorenzen $1952 b, 594)$
\end{abstract}

In the same year that "On the Consistency of the Concept of Infinity" is published, Lorenzen gives a talk at the second "Colloque International Logique Mathematique" in Paris. This talk is later published as Lorenzen 1954, where he provides a more detailed account of the notion of construct. It is especially interesting to see how he frames the introduction of constructs: it is to provide an answer to the challenge of eliminating the naive concept of set from analysis (p. 67). The notion of construct is explained, similarly to $1952 \mathrm{~b}, 591$, as a foundation for the basic notion of set $(1954,70)$. He then discusses how and why it could be legitimate to restrict the naive notion of set in such a way, and presents his construction of the language strata system, where each language stratum $S_{n}$, and indeed also $S_{\omega}$ and $S_{\omega+1}$, are constructs $(1954,71)$.

At this point it becomes quite clear that Lorenzen regards the concept of set as much more fundamental than the concept of infinity. When the concept of set is restricted to the acceptable notion of constructs, the concept of infinity indeed becomes consistent with the arguments presented in 1952b. But this is a consequence of settling the question of what a "good" concept of set is. So, the question of the right concept of infinity is secondary to the question of the correct concept of set; indeed the former is resolved by resolving the latter.

It only remains to be shown whether he still holds this position in the final version of operative mathematics in 1955. Here he does not use the notion of construct; instead, he replaces it with a more elaborate setup in which the key notion is definiteness.

The notion of definiteness (Definitheit) is very basic for operative logic and mathematics. In a nutshell, the sentence " $x$ is derivable" (or, respectively, " $x$ is underivable") is called definite because we know by the operationist system how to prove it (respectively, how to refute it). This can be called "definite via proof" (beweisdefinit) and "definite via refutation" (widerlegungsdefinit), respectively. He gives an inductive definition of definiteness already 
in the introduction of his book 1955, 5:

1. Every formula that is decidable via schematic operations is called definite.

2. If a notion of being definite via proof or being definite via refutation is determined for a formula, then the formula itself is called definite.

He then builds up the operative notion of set in the way we described in Section 3, always making sure that his logical and mathematical setup remains definite. This becomes particularly important when deciding where to terminate the construction of language strata. As we have seen above, Lorenzen is not settled on the exact point where the construction of language strata has to end; the only thing that is crucial for arriving at classical analysis is that one make the step to $S_{\omega}$ or $S_{\omega+1}$. However, for further according with Lorenzen's philosophical convictions, it is equally important that the construction does end at some point! $3^{\circ}$ The reason for the restriction of this iterative process is given in 1955, 189:

However, there would be no definite meaning in saying that the iteration should be continued "arbitrarily" long-in the same way, clauses from modern set theory, like the following, are not definite: "the index of the language strata should run through Cantor's II. number class (II. Zahlenklasse)".

So the notion of definiteness takes the place of the notion of construct in providing a boundary line for which sets should be permitted or excluded, as all operative sets have to appear in some language stratum. In a second step, this restriction of the concept of set then gives rise to the operative concept of infinity where uncountable infinities only appear relative to language strata.

We can therefore conclude that in the first of the proposed phases, Lorenzen indeed considered the concept of set the most fundamental philosophical notion in developing operative mathematics. ${ }^{31}$ His main motivation was to resolve the problems introduced by the classical notion of set. Again, this does not mean that he did not care about the question of actual-potential infinity. But it was not prominent in his foundational motivations; rather, the question of how to deal with the infinite gets resolved by using the "right" conception of set. We will see how this changed in the next subsection.

\subsection{Rejection of actual infinity}

To show Lorenzen's shifting of focus towards the concept of infinity in the second of the proposed phases, we will concentrate on the article "The

30 "... obviously not without boundaries, only up to the unproblematic ordinal numbers ..." (Lorenzen 1956a, 279).

${ }^{31}$ Again, this does not mean that he didn't have major mathematical motivations for the development of the operationist system (see also footnote 2). 
Actual-Infinite in Mathematics" (Lorenzen 1957). The reasons for considering only this article are twofold: First, the article gives a very clear formulation of the motivation behind his work in what we called the second phase. Especially the programmatic part at the end of the article constitutes what we claim to be the content of the second phase. Second, soon after the publication of this article, Lorenzen began to abandon operative logic and mathematics. According to Lorenz $(2001,35)$, the reason was a flaw in the concept of definiteness that ultimately made it inappropriate for Lorenzen's purposes. ${ }^{32}$ In his 1957, however, Lorenzen still considers operative mathematics, but this time motivated by eliminating the notion of actual infinity from mathematics.

This explains why the mathematical content of this paper is not new in comparison to his prior work. The philosophically motivated interpretation of this mathematical content, however, is novel, as Lorenzen looks at operative mathematics through the lens of the question of potential and actual infinity. Here we can find all of the direct references to these conceptions of infinity we were unsuccessfully looking for in the earlier works.

When introducing the construction of the natural numbers via the usual rules, Lorenzen states:

[To] assert that infinitely many such numbers really exist, that they can really be constructed by following this rule, would of course be false. ...

In philosophical terminology we say that the infinity of the number sequence is only potential; that is, it exists only as a possibility but does not actually (i.e., not in reality) exist. (Lorenzen 1987, 196; translated from Lorenzen 1957, 4-5)

He concludes that "in arithmetic there is ... no motivation to introduce the actual infinite" $(1987,197){ }^{33}$ He then discusses how actual infinity comes up in geometry and in the construction of the real numbers and finally discusses modern set theory:

It is this actual infinity of real numbers (latent in modern mathematics since the seventeenth century) that Cantor first brought explicitly to light, and it is the basis for the present acceptance of the Cantorian conception of infinity.

With the admission of the set of all real numbers as a legitimate object for mathematics there is simultaneously admitted the "power set," that is, the set of all subsets. (Lorenzen 1987, 200; translated from Lorenzen 1957, 8)

All of this is neither historically nor mathematically new. But Lorenzen frames this development completely under the motivational point of view of the actual-potential infinity debate. What consequences does he then draw for the future development of mathematics? He states:

32 "It was Alfred Tarski who ... convinced him of the impossibility to characterize arbitrary (logically compound) propositions by some decidable generalization of having a decidable proof-predicate or a decidable refutation-predicate. ... Hence, Lorenzen's attempt of an inductive definition of 'definite' in order to find a characterization of propositions which relinquishes the synonymy of 'definite' and 'decidably definite' had to be accepted as inappropriate" (Lorenz 2001, 35).

33 Translated from Lorenzen 1957, 5. 
The key to the indicated solution lies in replacing Cantor's power set of the set $C$ of all cardinal numbers with an appropriate potentially infinite set. ... Thus, instead of the power set, we have to construct an appropriate potentially infinite set of propositional forms. (Lorenzen 1987, 201; translated from Lorenzen 1957, 10)

This is the locus where the proposed shift truly comes into play. Lorenzen still concludes that the concept of (a certain kind of) set has to be replaced by a different notion, but the motivation, explicitly stated, is to eliminate actual infinity. The replacement of the concept of set now becomes merely the way in which such a reduction to potential infinity is carried out. The new concept of set has to fulfill the requirement of being only potentially infinite; so, here, the concept of infinity is the primary concern; the concept of set is secondary. Lorenzen states this quite clearly in the programmatic appeal with which he concludes the article:

If the conception developed here is correct, it represents for modern mathematics a reform ....

Just as at that time the infinitely small was to be eliminated from mathematics, now also the infinitely large (more precisely, the actual infinite) is to be demonstrated to be dispensable. (Lorenzen 1987, 201-202; translated from Lorenzen 1957, 10-11)

\section{Conclusion and outlook}

We have shown above that Lorenzen shifts his focus from considering the concept of set as the more fundamental notion in his foundational work in operative mathematics, until the mid-5os, to focusing on the concept of the infinite and, in particular, the actual-potential infinity distinction in the later 1950s. This seems to indicate that Lorenzen's operative work was not simply a precursor for his later work, but a body of work with its own philosophical and mathematical motivations.

As mentioned before, two questions still remain open: First, why does this shift occur? And secondly, regardless of Lorenzen's motivations, does operative mathematics represent a framework for potential infinity? The answers to both questions require more work than can be done within the scope of this article; so let us just point out some considerations towards possible answers.

Regarding the question of why such a shift occurred, a simple answer presents itself. The 1950 os marked a significant change for Lorenzen from an institutional point of view. Having been appointed a professor of mathematics at the University of Bonn in 1952, he changed to a professorship in philosophy at the University of Kiel in 1956. From a thematic point of view, this may not seem surprising, since he had been working on the foundations of mathematics for some years. Nevertheless, Lorenzen perceived it as a change himself, remarking upon it several times in correspondence with the 
philosopher Oskar Becker (who was Lorenzen's colleague in Bonn), as in this slightly sarcastic passage:

Besides, as I am a "philosopher" now, this is all right with me, because it is common with "philosophical" books that they are completely misunderstood. (Letter from Lorenzen to Becker, August 22nd, 1957, OB 5-1-1, Philosophical Archive, University of Konstanz) ${ }^{34}$

So, one explanation for this shift is that the context of debate changed. One could argue that during the first phase, Lorenzen primarily wanted to inform the mathematical debate on foundational issues, as it developed in the aftermath of the so-called foundational crisis. In the second phase, however, Lorenzen re-framed his operationist approach to inform the philosophical debate about mathematical infinity. To fully argue for such a thesis, more work has to be done. But, reading Lorenzen's papers, one can always find hints that he was very aware of which discussions would be considered interesting for the mathematical community and its discourse on foundational issues, and which would be better suited for the philosophical community. 35 So, this is a viable candidate for an explanation of this shift.

The systematic question of whether the operationist approach is indeed a framework for potential infinity (and does not assume any kind of actual infinity) has no clear answer from the outset. Obviously, Lorenzen considered this to be the case, as he names operative mathematics as one way of replacing the usual power set with a potential infinity of formulas (Lorenzen 1957, 10). But this view did not remain uncontested. Niebergall (2004) analyzes the underlying assumption of infinity Lorenzen draws from in setting up basic numbers (via the usual rules presented in operative mathematics) and thoroughly examines Lorenzen's notions of "always-counting-on" and, more generally, rule-based infinite processes (see Niebergall 2004, 171). In the end he concludes that such approaches to infinity can never stay merely potential; one always has to assume some kind of actual infinity, "be it of infinite objects or be it of infinitely many objects" (Niebergall 2004, 171). ${ }^{36}$

Looking at operative mathematics, this analysis seems to hold even more strongly. Let us consider the fundamental framework in which operative analysis is developed, namely the framework of language strata. The fact that the first language stratum contains infinitely many objects can be explained

34 "Da ich jetzt ja außerdem ,Philosoph' bin, ist mir das auch insofern recht, weil es bei ,philosophischen' Büchern ja gang und gäbe ist, daß sie völlig mißverstanden werden."

35 One such occurrence is the following quote (more can easily be found in his papers): "Taking the cavalier attitude that most mathematicians display toward philosophical questions, we could try simply to ignore these 'sophistical' problems" (Lorenzen 1987, 197-198; translated from Lorenzen 1957, 6).

${ }^{36}$ This conclusion can be contested, though: one of the referees pointed out that Niebergall's critique only seems to hold when reconstructing Lorenzen's approach in standard set theory, and this is not the way Lorenzen intended it to be read. It would be interesting to contrast these different viewpoints in more detail and to investigate to what extent modern analyses (like the one of Linnebo and Shapiro 2019) can have an impact on Lorenzen's notion of potential infinity. 
by the rule-based procedures of schematic operations (although this is exactly what Niebergall criticizes). However, the most important step in the construction of the language strata occurs when defining $S_{\omega}$ and $S_{\omega+1}$. As we have seen, $S_{\omega}$ is the union of all previous language strata. Note that this does not yet mean that we are actually building a new language over atoms in this step. This only happens when we define $S_{\omega+1}$. There, we consider the infinite union of all of the infinite sets in the $S_{n}$, and in $S_{\omega+1}$ we begin to add new objects to this infinite union of infinite objects. Thus, we now not only allow sets whose elements can be defined by a construction process, but we have to consider infinitely many different construction processes at once (to know if an $x$ is in $S_{\omega}$ we have to search through infinitely many $S_{n}$ and check if $x$ appears in one of them). Lorenzen states that allowing such procedures is motivated by mathematical expediency:

If we want to attain that every set also appears as an element, we have have to go
up to a limit ordinal with the index of the language strata. For example, one can
go beyond the language strata with finite index by building a set whose elements
are $u,\{u\},\{\{u\}\}, \ldots$. This set is only representable in $S_{\omega+1}$. (Lorenzen 1955,
190)

As Lorenzen $(1955,189)$ himself remarks, this step is crucial for mirroring classical analysis in operative mathematics. Even if Niebergall's abovementioned arguments could be sidestepped, the setup with language strata seems to make it difficult to argue that operative mathematics provides a framework for potential infinity.

Perhaps a solution to this difficulty can be found in recent work on actual and potential infinity by Linnebo and Shapiro (2019). Here the authors address Niebergall's general claim that there is no clear way of expressing potential infinity without either staying finite or falling back on actualist assumptions about infinity (see Linnebo and Shapiro 2019, 166). They propose a way out of Niebergall's dilemma by using modal logic to explicate different potentialist positions. Indeed, they are able to show that there is a way of being potentialist about infinity while still using classical logic (instead of having to use intuitionistic logic). It could be an interesting future project to see how Lorenzen's operationist system, or indeed similar constructive approaches, fares when analyzed by means of Linnebo and Shapiro's modal explication.

\section{References}

Bernays, Paul. 1952. Review: "Über endliche Mengen" by Paul Lorenzen. Journal of Symbolic Logic 17 (4): 275-276.

Coquand, Thierry, and Stefan Neuwirth. 2017. "An introduction to Lorenzen's 'Algebraic and logistic investigations on free lattices' (1951)." arXiv:1711.06139 [math.LO]. 
- 2020. "Lorenzen's proof of consistency for elementary number theory." History and Philosophy of Logic, to appear. doi:10.1080/0144 5340.2020 .1752034

Craig, William. 1957. Review: Einführung in die Operative Logik und Mathematik by Paul Lorenzen. Bulletin of the American Mathematical Society 63 (5): 316-320.

Ferreirós, José. 2007. Labyrinth of thought: A history of set theory and its role in modern mathematics. Basel: Birkhäuser.

Fraenkel, Abraham A., Yehoshua Bar-Hillel, and Azriel Levy. 1973. Foundations of set theory. Volume 67 of Studies in Logic and in the Foundations of Mathematics. Amsterdam: Elsevier.

Frey, Gerhard. 1957. Review: Paul Lorenzen: Einführung in die operative Logik und Mathematik. Zeitschrift für philosophische Forschung 11:631632.

Heyting, Arend. 1957. Review: Paul Lorenzen, Das Aktual-Unendliche in der Mathematik; Paul Lorenzen, Die Rolle der Logik in der Grundlagenkrisis der Analysis. Journal of Symbolic Logic 22 (4): 368.

Kahle, Reinhard, and Isabel Oitavem. 2020. "Lorenzen between Gentzen and Schütte." In this volume, pages 61-73.

Linnebo, Øystein, and Stewart Shapiro. 2019. "Actual and potential infinity." Noûs 53 (1): 160-191.

Lorenz, Kuno. 2001. "Basic objectives of dialogue logic in historical perspective." Synthese 127:255-263.

Lorenzen, Paul. 1951a. "Die Widerspruchsfreiheit der klassischen Analysis." Mathematische Zeitschrift 54:1-24.

_. 1951b. "Konstruktive Begründung der Mathematik." Mathematische Zeitschrift 53:162-202.

_. 1951C. "Maß und Integral in der konstruktiven Analysis." Mathematische Zeitschrift 54:275-290.

—. 1952a. "Über den Mengenbegriff in der Topologie." Archiv der Mathematik 3:377-386.

—. 1952b. "Über die Widerspruchsfreiheit des Unendlichkeitsbegriffes." Studium Generale 5:591-594.

338.

. 1952c. "Über endliche Mengen." Mathematische Annalen 123:331-

. 1954. "Die Rolle der Logik in der Grundlagenkrisis der Analysis." In Applications scientifiques de la logique mathematique: Actes de $2^{e}$ Colloque International Logique Mathematique, Paris 25-30 aout 1952, Institut Henri Poincaré, pages $65-73$.

- 1955. Einführung in die operative Logik und Mathematik. Springer.

—. 1956a. "Die Fiktion der Überabzählbarkeit." In Proceedings of the International Congress of Mathematicians 1954, Amsterdam September 2 September 9, volume III, pages 273-279. North-Holland.

—. 1956b. "Über den ,Operativismus'." Unpublished document.

—. 1957. "Das Aktual-Unendliche in der Mathematik." Philosophia 
Naturalis 4 (1): 1-11.

. 1960. "Constructive and axiomatic mathematics." Synthese 12 (1):

114-119.

- 1987. "The actual-infinite in mathematics." Translation by K. R. Pavlovic of Lorenzen 1957. In his Constructive philosophy, pages 195-202. Amherst: University of Massachusetts Press.

Niebergall, Karl-Georg. 2004. "Is ZF finitistically reducible?" In One hundred years of Russell's Paradox: Mathematics, logic, philosophy, edited by G. Link, pages 153-180. Berlin, New York: Walter de Gruyter.

Schroeder-Heister, Peter. 2008. "Lorenzen's operative justification of intuitionistic logic." In One hundred years of intuitionism (1907-2007): The Cerisy conference, edited by M. van Atten, P. Boldini, M. Bourdeau, and G. Heinzmann, pages 214-240. Basel: Birkhäuser.

Sieg, Wilfried. 1999. "Hilbert's programs: 1917-1922." Bulletin of Symbolic Logic 5 (1): 1-44.

Skolem, Thoralf. 1957. Review: Paul Lorenzen, Einführung in die operative Logik und Mathematik. Journal of Symbolic Logic 22 (3): 289-290.

Stegmüller, Wolfgang. 1958. Review: Paul Lorenzen, Einführung in die operative Logik und Mathematik. Philosophische Rundschau 6 (3/4): 161-182. Weyl, Hermann. 1918. Das Kontinuum. Leipzig: Veit \& Co.

Open Access This chapter is licensed under the terms of the Creative Commons Attribution 4.0 International License (http://creativecommons.org/licenses/by/4.0/), which permits use, sharing, adaptation, distribution and reproduction in any medium or format, as long as you give appropriate credit to the original author(s) and the source, provide a link to the Creative Commons license and indicate if changes were made.

The images or other third party material in this chapter are included in the chapter's Creative Commons license, unless indicated otherwise in a credit line to the material. If material is not included in the chapter's Creative Commons license and your intended use is not permitted by statutory regulation or exceeds the permitted use, you will need to obtain permission directly from the copyright holder. 